\title{
Fístula peritoneocutánea por cálculos biliares. Una complicación tardía de la colecistectomía laparoscópica*
}

\author{
Drs. RODRIGO IGLESIS G. ${ }^{1}$, KUEN LEE CH. ${ }^{1}$, JUAN M. DONAIRE A. ${ }^{1}$, NICOLÁS KERRIGAN B. ${ }^{1}$ \\ 1 Servicio de Cirugía, Hospital Militar, Santiago, Chile.
}

\begin{abstract}
Peritoneocutaneous fistula caused by abandoned biliary stones during laparoscopic cholecystectomy
\end{abstract}

Gallbladder perforation may occur during laparoscopic cholecystectomy. The leakage of bile and stones to the peritoneal cavity is less common and the real incidence of abandoned stones is hard to ascertain. The possible complications of these abandoned stones are not well known. We report five patients (four females) aged 49 to 78 years, with a peritoneo-cutaneous fistula caused by biliary stones abandoned in the peritoneal cavity during laparoscopic cholecystectomy performed between 1.5 and 10 years before. We conclude that abandoned stones in the peritoneal cavity can be associated to important complications and those that leak to the peritoneal cavity during laparoscopic cholecystectomy, should be removed during the surgical procedure and the gallbladder should be excised in a bag. The conversion to open surgery for an exhaustive peritoneal lavage is apparently not indicated.

Key words: Peritoneocutaneous fistula, biliary stones, laparoscopic cholecystectomy.

\section{Resumen}

La perforación de la vesícula biliar ocurre con alguna frecuencia durante la colecistectomía laparoscópica (CL). El derrame de bilis y cálculos en la cavidad peritoneal es menos frecuente y la verdadera incidencia de cálculos abandonados en ésta cavidad es difícil de determinar. Éstos no son considerados un peligro; sin embargo, la exacta biología natural de estos cálculos extraviados no está bien dilucidada y parece ser que no son tan inocuos como se creía, ya que con frecuencia se están reportando variadas complicaciones tardías de esta condición. Presentamos 5 pacientes atendidos en nuestro servicio por fístula peritoneocutánea secundaria a litiasis biliar abandonada en la cavidad abdominal durante la CL practicada entre 1,5 y 10 años atrás. Concluimos que, si bien es cierto que las complicaciones secundarias a cálculos biliares abandonados en la cavidad peritoneal son poco frecuentes $(0,08-0,3 \%)$, cuando ocurren se traducen en alta morbilidad, por lo que debe evitarse esta condición retirando todos los cálculos derramados al ocurrir una rotura vesicular, e idealmente exteriorizarla en una bolsa. La conversión a cirugía abierta para aseo peritoneal exhaustivo pareciera no estar indicada, pero si la necesidad de entregar clara

*Recibido el 20 de Diciembre de 2007 y aceptado para publicación el 17 de julio de 2008.

Correspondencia: Dr. Nicolás Kerrigan B.

Holanda 050, Santiago, Chile

E-mail: nicokerrigan@gmail.com 
información al paciente acerca de las potenciales complicaciones tardías que la situación puede conllevar, evitando así problemas médico legales futuros.

Palabras clave: Colecistectomía laparoscópica, rotura vesicular, cálculos abandonados, fístula peritoneo-cutánea.

\section{Introducción}

La perforación de la vesícula biliar ocurre frecuentemente durante la colecistectomía laparoscópica (CL) dándose cifras entre 10 y $40 \%{ }^{1-4}$. El derrame de bilis y cálculos en la cavidad peritoneal es menos frecuente; el encontrarlos y retirarlos suele ser extremadamente laborioso, y por lo general la tarea es abandonada en la creencia que estos son poco dañinos. La verdadera incidencia de cálculos sembrados en la cavidad abdominal es difícil de determinar. Algunas series reportan entre 6 y $33 \% 5,6$; sin embargo, la exacta biología natural de estos cálculos y su efecto a largo plazo aún no han sido dilucidados, y cada día se reportan nuevas y severas complicaciones derivadas de ésta situación. Presentamos nuestra experiencia en el enfrentamiento de 5 casos de una de estas complicaciones, la fístula peritoneocutánea secundaria a litiasis biliar abandonada en la cavidad abdominal.

\section{Material y Método}

La serie retrospectiva esta formada por 5 pacientes atendidos en nuestro servicio entre mayo de 1992 y marzo de 2006 por presentar una fístula peritoneocutánea secundaria a cálculos biliares abandonados durante una CL. La CL había sido practicada entre 1,5 y 10 años antes de la atención de la que damos cuenta.

\section{Resultados}

Caso 1. ANF, mujer de 70 años. Antecedente de CL 3 años antes. Consultó por dolor y aumento volumen lumbar derecho de $6 \mathrm{~cm}$. La ecografía y la TAC informaron una lesión de aspecto granulomatoso en el espesor del músculo oblicuo, sin poder descartar un proceso neoplásico. En las imágenes no se observan anormalidades intraperitoneales. La punción de la masa para citología fue negativa para neoplasia. La cirugía reveló una lesión abscedada, con un trayecto fistuloso hasta el peritoneo en la región de la fosa iliaca derecha que contenía múltiples cálculos facetados de tipo vesicular. Evoluciona con fístula crónica en relación a la cicatriz, la que cerró a los 18 meses. Dos años después presenta nuevo absceso de ubicación submamaria derecha, con eliminación de nuevos cálculos. Rechaza otra cirugía y la fístula cierra espontáneamente dos años después. No ha presentado a la fecha nuevas reactivaciones.

Caso 2. AOM, hombre de 78 años. Antecedente de CL 18 meses antes. Consulta por baja de peso y fiebre. El estudio ecográfico demostró una colección supurada retrohepática adherida a la pared abdominal. Se realiza un drenaje percutáneo bajo ecografía, con mejoría del cuadro clínico. Reingresa 2 meses más tarde por un cuadro similar con una fístula en el sitio de punción anterior. La TAC identifica la misma colección, con una imagen cálcica de un $\mathrm{cm}$ en el centro y un trayecto fistuloso hacia la pared posterior. Se extrajo un cálculo por laparotomía subcostal con mejoría completa del paciente.

Caso 3. BBM, mujer de 65 años. Antecedente de CL 3 años antes. Dolor lumbar de larga data, hasta que se drenó un absceso lumbar derecho espontáneamente y evolucionó con fístula crónica. La ecografía no reveló anormalidades. Se practicó una fistulectomía, comprobando un trayecto que entraba a la cavidad abdominal en la fosa iliaca derecha, en la que se encontró un cálculo de tipo biliar.

Caso 4. GGP, mujer de 57 años. Antecedente de CL hace 10 años. Evoluciona con dolor lumbar inespecífico de larga data y aparición de una fístula lumbar, que fue resecada por un dermatólogo, con reaparición al cabo de un mes. La TAC reveló un proceso fibrótico suprahepático sugerente de hematoma subcapsular antiguo. Se realiza una fistulectomía, llegando a la cavidad y comprobando un proceso adherencial suprahepático que contenía en su interior múltiples cálculos biliares (Figuras 1, 2, 3 y 4$)$.

Caso 5. ASG, mujer de 49 años. Antecedente de CL 4 años antes. Dolor umbilical de varios meses de evolución, con 2 episodios separados de abscesos umbilicales con expulsión de cálculos. Permanece con una fístula crónica. Las imágenes no revelaron anormalidades intraperitoneales. Se realizó una onfalectomía, comprobando la presencia de un 


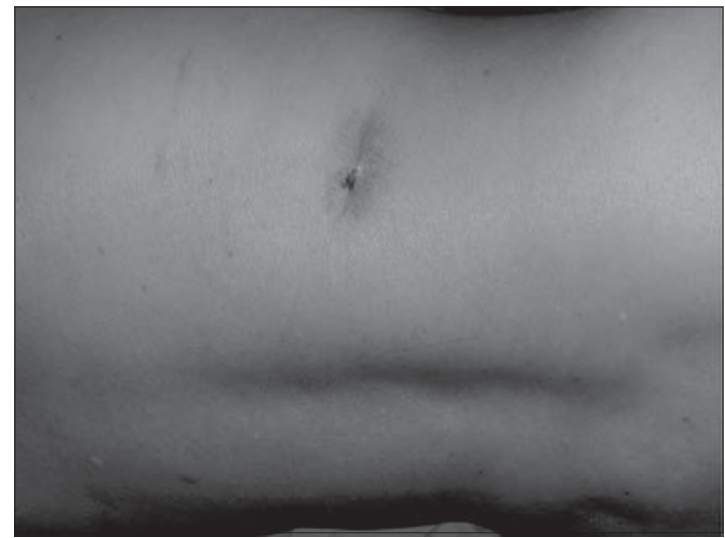

Figura 1. Fístula peritoneo cutánea crónica en paciente colecistectomizado.

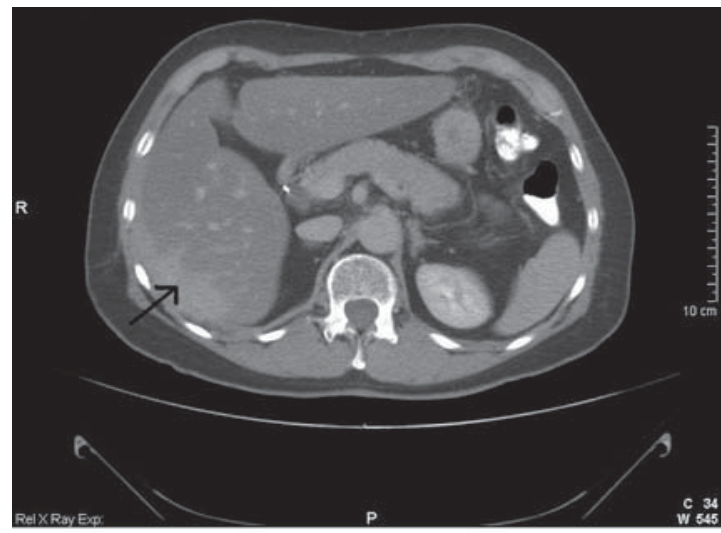

Figura 3. TAC abdominal que muestra zona informada como hematoma subcapsular del hígado.

trayecto hacia el peritoneo en el interior de un proceso adherencial interasas, con litiasis en su interior.

En la totalidad de los pacientes los informes de anatomía patológica informaron la presencia de una fístula inespecífica.

\section{Discusión}

En los últimos años la CL se ha convertido en el tratamiento de elección de la litiasis biliar no complicada. Esta nueva era de cirugía laparoscópica nos ha enfrentado a inesperadas complicaciones tardías que no eran vistas con la colecistectomía abierta ${ }^{7}$.

Como el evento intraoperatorio más frecuente se ha descrito con la CL la ocurrencia de una perfora-

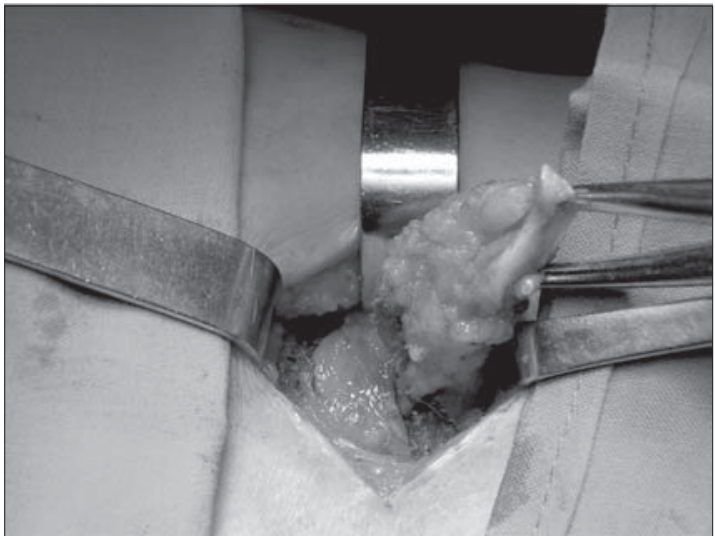

Figura 2. Fistulectomía.

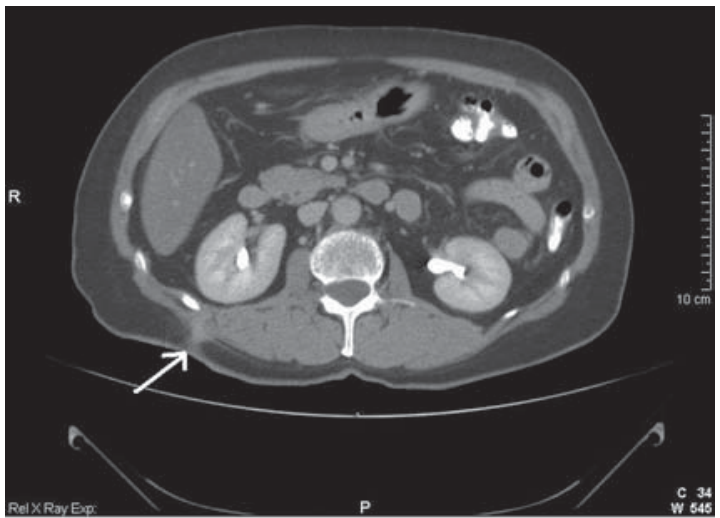

Figura 4. TAC que muestra fístula lumbar.

ción de la vesícula biliar. Esta puede ocurrir cuando esta siendo separada del lecho hepático, o bien cuando se exterioriza por uno de los sitios de punción. La perforación vesicular, el derrame de bilis y cálculos en la cavidad peritoneal ocurren hasta en un tercio de los pacientes ${ }^{6}$, aunque su real frecuencia es difícil de determinar. El aseo, y en forma especial la extracción de este material es laborioso y tedioso, lo que hace que por lo general los cálculos sean abandonados por el cirujano, en la creencia que estos son inocuos. Así lo han demostrado estudios en animales ${ }^{7-10}$, aunque algunos sugieren que aumentarían significativamente la formación de adherencias y la posibilidad de abscesos ${ }^{11,12}$. Gurleyik y cols, concluyen que la composición química de los cálculos jugaría un rol determinante. Así, aquellos con pigmentos producirían más reacción que los de colesterol ${ }^{13}$. La exacta biología na- 
tural de este material en el peritoneo en el largo plazo no ha sido dilucidada hasta el momento. Estudios clínicos retrospectivos y prospectivos realizados para determinar las reales consecuencias del abandono de los cálculos en la cavidad peritoneal han demostrado que la mortalidad y la incidencia de complicaciones serias es baja $(0,08$ a $0,6 \%)$, no aconsejando la conversión y aseo por laparotomía $^{14,15}$. Sin embargo, se reportan en la literatura casos aislados de las más variadas formas de presentación de cálculos abandonados en el peritoneo desde 1 mes hasta 20 años post cirugía (Tabla 1$)^{16-36}$.

La forma poco común de presentación de la litiasis biliar abandonada como fístula cutánea, no suele ser sospechada en el preoperatorio. Como sucedió en nuestra serie en los 3 primeros pacientes, en los que el primero fue diagnosticado como un proceso neoplásico muscular, y los dos siguientes como fístulas de etiología no precisada hasta que se encontraron en el intraoperatorio cálculos biliares, lo cual es coincidente con lo que ha sido reportado en la literatura. Probablemente esta situación no sucedería si el cirujano tuviera en mente la posibilidad de esta complicación tardía, informara a su pa-

Tabla 1. Complicaciones secundarias a cálculos biliares abandonados ${ }^{16-36}$

Infecciosas
Local
Absceso hepático
Absceso subhepático
Absceso retrohepático
Absceso intrabdominal
Distante
Absceso retroperitoneal
Absceso lumbar
Absceso pélvico
Complicaciones cutáneas
Formación de cavidad
Infección del sitio de entrada
Formación de granuloma
Fístula colocutánea
Mecánicas
Obstrucción intestinal
Depósito en saco herniario
Migración
Pélvica (dispareunia, tenesmo vesical)
Tórax (empiema, coleliptosis)
Tracto urinario (excreción, hematuria)
Sistémica
Sepsis

ciente en el post operatorio y lo siguiera estrictamente a largo plazo.

El estudio, una vez presentada la complicación, incluye ecografía, TAC y eventualmente una fistulografía. Tal como sucedió en nuestros pacientes, y así también lo reporta la literatura, no siempre las imágenes demuestran la litiasis abandonada. El tratamiento consiste en la extracción de los cálculos mediante una fistulectomía o con instrumentación (Canastillo de Dormia, nefroscopio, u otro). En nuestra serie todos fueron operados, y una de ellos recidivó en otro sitio cutáneo años después, lo que sugiere una dispersión de los cálculos en la cavidad peritoneal con el lavado y el neumoperitoneo operatorios.

\section{Conclusiones}

La fístula peritoneocutánea es una complicación tardía, que puede ocurrir cuando se han abandonado cálculos en el peritoneo durante la CL. Su frecuencia es baja pero de morbilidad considerable y que puede acarrear consecuencias médico legales.

Se sugiere que el manejo apropiado de la siembra peritoneal de cálculos biliares durante la CL debe incluir una información preoperatoria al paciente que la situación puede ocurrir dependiendo de las condiciones de la vesícula biliar y del tamaño y número de cálculos. Cuando sucede el evento en el intraoperatorio, se debe extraer la vesícula en bolsa y desplegar todo el esfuerzo posible intentando el retiro de los cálculos con un aseo prolijo de la cavidad (no está indicada la laparotomía), así como consignar claramente en el protocolo operatorio la ocurrencia del hecho, informar al paciente y seguirlo estrechamente en el post operatorio.

\section{Referencias}

1. Schafer M, Suter C, Klaiber C. Spilled gallstone after laparoscopic cholecistectomy. A relevant problem? A retrospective analysis of 10.174 laparoscopic cholecistectomies. Surg Endosc 1998; 12: 291-293.

2. Memon MA, Deeik RK, Maffi TR, Fitzgibbons RJ Jr. The autcome of unretrieved gallstone in the peritoneal cavity during laparoscopic cholecistectomy. A prospective analysis. Surg Endosc 1999; 13: 848-857.

3. Diez J, Arozamena C, Gutiérrez L. Lost stone during laparoscopic cholecistectomy. HPB Surg 1998; 11: 105-108; discussion 108-109.

4. Rice DC, Memon MA, Jamison RL. Long term consequences of intraoperative spillage of bile and gall stones during laparoscopic cholecistectomy. J 
Gastrointest Surg 1997; 1: 85-91.

5. Catarci M, Zaraca F, Scaccia M, Carboni M. Lost intraperitoneal stones alter laparoscopic cholecistectomy: harmless secuela or reason for reoperation? Surg Laparosc Endosc 1993; 3: 318-322.

6. Fitzgibbons RJ, Annibaldi R, Litke Bs. Gallblader perforation and gallstone removal: open versus laparoscopy and pneumoperitoneum. Am J Surg 1993; 165: 497-504.

7. Schirmer BD, Winters KL, Edlich RF. Cholelithiasis and cholecistitis. J Long Term EFF Med Impleants 2005; 15: 329-338.

8. Welch N, Hinder RA, Fitzgibbons R. Gallstones in the peritoneal cavity: a clinical and experimental study. Surg Laparos 1997; 7: 103-112.

9. Cohen RV, Pereira PR, Barros MV. Is the retrieval of lost peritoneal gallstone worthwhile? Surg Endosc 1994; 8: 1360.

10. Johnston S, McEntee $\mathrm{G}$. The need to retrieve the dropped stone during laparoscopic cholecistectomy. Am J Surg 1994; 167: 608-610.

11. Leland DG, Dawson DL. Adhesion and experimental intraperitoneal gallstone. Contemp Surg 1993; 42: 273-276.

12. Tzardis PJ, Vougiouklakis D, Lymperi M. Septic and other complications resulting from biliary stones placed in the abdominal cavity: experimental study in rabbits. Surg Endosc 1996; 10: 553-556.

13. Gurleyick E, Gurleyick G, Yucel O, Unalmiser S. Does chemical composition have an influence on the fate of intraperitoneal gallstone in rat? Surg Laparosc Endosc 1998; 8: 113-116.

14. Soper NJ, Dunnegan DL. Does intraoperative gallbladder perforation influence the early outcome of laparoscopic cholecistectomy? Surg Laparosc Endosc 1991; 1: 156-161.

15. Rice DC, Memon MA, Jamison RL. Long-term consequences of intraoperative spillage of bile and gallstone during laparoscopic cholecistectomy. J Gastrointest Surg 1997; 1: 85-91.

16. Sterman PH, Sterman SN. Unretrieved gallstone presenting as a Streptococcus bovis liver absces. Journal of the Society of Laparoscopic Surgeons 2000; 4: 263 265.

17. VanBrunt PH, Lanzafrane RJ. Subhepatic inflamatory mass after laparoscopic cholecistectomy. Arch Surg 1994; 129: 882-883.

18. Grestchel S, Engelmann L, Estevez-Schwarz M. Wolf in sheeps clothing: spilled gallstones can cause severe complications aftyer endoscopic surgery. Surg Endosc 2001; 15: 98-101.

19. Mellinger JD, Eldrige TJ, Eddelman ED. Delayed gallstone absces following laparoscopic cholecistectomy. Surg Endosc 1994; 8: 1332-1334.

20. Parra E, Munshi IA, Armstrong JH, Sleman D, Levi JU. Retroperitoneal absces as a complication of retained gallstone following laparoscopic cholecistectomy. J Laparoendosc Adv Surg Tech A 1998; 8: 89-93.

21. Galinaro RN, Miller FB. The last gallstone. Complication after laparoscopic cholecistectomy. Surg Endosc 2003; 17: 159.

22. Protopapas A, Milingos S, Diakomanolis E. Septic lithiasis of the pelvis. Surg Endosc 2003; 17: 159.

23. Cacdac RG, Lakra YP. Abdominal wall sinus tract secondary to gall stones. A complication of laparoscopic cholecystectomy. J Laparoendosc Surg 1993; 3: 509-511.

24. Yao CC, Wong HH, Yang CC. Abdominal wall abcess secondary to spilled gallstones: late complication of laparoscopic cholecystectomy and preventive measures. Laparoendosc Adv Surg Tech A 2001; 11: 47-51.

25. Golub R, Nwogu C, Cantu R. Gallstone shrapnel contamination during laparoscopic cholecystectomy. Surg Endosc 1994; 8: 898-900.

26. Patterson EJ, Nagy AG. Don't cry over spilled stones? Complications of gallstones spilled during laparoscopic cholecystectomy: case report and literatura review. Can J Surg 1997; 40: 249-250.

27. Tekin A. Mechanical small bowel obstruction secondary to spilled stones. J Laparoendosc Adv Surg Tech A 1998; 8: 157-159.

28. Rosin D, Korianski Y, Yudich A. Lost gallstones found in a hernial sac. J Laparoendosc Surg 1995; 5: 409-411.

29. Chanson C, Nassiopoulos K, Petropoulos P. Complications of intraperitoneal gallstones. Schweiz Med Wochenschr 1997; 127: 1323-1328.

30. Pfeifer ME, Hansen KA, Tho SP. Ovarian Cholelithiasis alter laparoscopic cholecystectomy associated with chronic pelvis pain. Fértil Steril 1996; 66: 1031-1032.

31. Downie GH, Robbins MK, Souza JJ. Cholelithoptysis: a complication of laparoscopic cholecystectomy. Chest 1993; 103: 616-617.

32. Kelty CJ, Thorpe JA. Empyema due to spilled stones during laparoscopic cholecystectomy. Eur J Cardiothorac Surg 1998: 14: 445-446.

33. Castro MG, Alves AS, Oliveira CA. Elimination of biliary stones through the urinary tract: a complication of the laparoscopic cholecystectomy. Rev Hosp Clin Fac Med Sao Paulo 1999: 54: 209-212.

34. Famulari C, Pirrone G, Macri A. The vesical granuloma: rare and late complication of laparoscopic cholecystectomy. Surg Laparosc Endosc Percutan Tech 2001; 11: 368-371.

35. Bruggermeyer MT, Saba AK, Thibodeaux LC. Absces formation following spilled gallstone during laparoscopic cholecistectomy. Journal of the Society of the Laparoendoscopic Surgeon 1997; 1: 45-52.

36. Tumer AR, YuksekYN, Yasti CY. Dropped gallstone during laparoscopic cholecistectomy: The consequences. World J Surg 2005; 29: 437-440. 